\title{
Public Health Schools in India Need to Go Beyond Syllabus
}

\section{Suresh $\mathrm{K}^{*}$}

Public Health Consultant, India

*Corresponding author: Suresh Kishanrao, Public Health Consultant, Bengaluru, India, Tel: 919810631222; Email: ksuresh.20@gmail.com

\section{Research Article}

Volume 3 Issue 2

Received Date: March 10, 2020

Published Date: April 06, 2020

DOI: $10.23880 /$ jqhe-16000155

\section{Abstract}

What tops the priority chart for recruiters in Public Health in India today? Skills of Field epidemiology, working under local constraints, out-off box innovation, client centric approaches in hospitals management, business attitude and aptitude, presentation and communication. The ancient Indian system of education had an integrated approach to training of head (mind), hand (skills), and heart (human values and ethics). However, most of the present-day MPH courses are designed to expose candidates to 5 core discipline areas of public health-Biostatistics, Epidemiology, Environmental Health, Health Policy and Health Administration and Social and behavioural sciences. It goes without saying that Public Health Management education must fall in line with almost all other disciplines, where there is a conscious effort to align not only with the evolving technology but also business dynamics.

Teaching scholars about the feelings of others lays the foundation for strong interpersonal skills that will be invaluable in their professional and personal lives. To address the widening skill-gap, primarily introducing industry exposure and latest trends in the filed epidemiology, vaccinology, national health programs and health facilities management at different levels of the local health system is the need of the time. Secondly the classroom experiences need to evolve from scholars competing for grades to scholars working together learning to form friendships and deal with complex social interactions. For students, it may be a touch difficult to zero in on a skill set that would stand them in good stead. Faculty must know the ground reality and students should be exposed to it directly. Counselling and mentoring play a crucial role here.

The newer Public Health recruitment demands have got to enter the syllabus of the mainstream master's in Public Health courses if any country wants to prepare students for the future that awaits them. In moderns Public Health postgraduate courses whenever a theory is taught, it must be related to something in the real world or from real time data. Even in the exit examinations, theory questions and practical's must be from the real world. Teaching methodologies must look at going beyond the pedagogical lectures, books, presentations and internet search to designing a curriculum that offers holistic education.

Keywords: Public Health Industry; Syllabus; Skill Gap

Abbreviations: MPH: Master's in Public Health; PGDHM: Postgraduate Diplomas in Health and Hospital administration; UGC: University Grant's Commission; MOH\&FW; Ministry of Health \& Family Welfare; AIPH: India Institute of Public Health Kolkata; DIH: Diploma in Industrial Health; NIMHANS: National Institute of Mental Health and Neurosciences; NGO: Nongovernmental Organization.

\section{Introduction}

The ancient Indian system of education had an integrated approach to training of head (mind), hand (skills), and heart (human values and ethics). Gandhi came out with a new model of education and called it Nai Talim (new education), known as Basic Education. To Gandhi, the objective of education was the harmonious development of the individual, drawing out 
the best in him. To him, the body is the means of earning a living; mind is the means of reasoning, while the soul is a place for building character. The learner needs to understand the importance of education for earning a living, for acquiring knowledge, and for building a loving personality [1].

UNICEF is driven by five core values-care, respect, integrity, trust and accountability. A range of initiatives aimed at strengthening its commitment to its core values include: i) Promoting a 'speak-up culture' to help create a safer and more inclusive workplace. ii) Seeking expert guidance on where it is not yet successful and where it could do better iii) Implementing measures towards a culture of gender equality in the workplace to achieve. UNICEF believes in capacity building of their staff to be skilled in thinking by head (conceptualization), soiling their feet (working with unreached or under-reached) for their ability to put hands in other's (philanthropists) pockets (fund rising) [2].

When studying for an MPH degree scholar is expected to develop an integrated interdisciplinary, cross-cutting set of overall competency domains like Health and Hospital management, Communication and Informatics, Diversity and Culture, Rural / urban Immersion, Leadership, Professionalism, Program Planning, Epidemiology, National Health Programs, Health System, National Health Policy, International health Public Health Biology, and Systems Thinking [3].

However, I have observed since 1976 when I did my post graduate Diploma in Industrial Health (DIH) that most of our traditional MPH degrees are designed to expose candidates to 5 core discipline areas of public health-Biostatistics, Epidemiology, Environmental Health, Health Policy and Health Administration and Social and behavioural sciences. Most of the teachers work hard to complete the syllabus in words but not in spirit. Books / pedagogical teaching/ learning/ Library-that is synonymous for what we got in most of the universities and institutions. Limited practical exposure, very little skill building and by no means a holistic education that focused on developing all the aspects of a human being and not just the academic mastery was the norm. India used to give public health postgraduate diplomas (DPH) and degrees (MD (P\&SM) to only basic allopathic medical graduates (MBBS) until 1995. It was only since 1995 MPH courses are open to non-allopathic graduates and even non-medical graduates. The true purpose of education 'is to make minds not careers' says a popular proverb. Being a good student in the school has nothing to do with success or happiness in life. We find that our education has not prepared us for daunting challenges, difficult emotional or social experiences.

Master of Public Health programs are available in India through Medical Colleges, Public Health Schools, Colleges of Allied Sciences and specialized National institutions of Public Health like National Institute of Mental Health and Neurosciences (NIMHANS) Bengaluru, National Institute of Epidemiology, Chennai(NIE), National Centre for Disease Control (NCDC) Delhi, National Institute of Health \& Family Welfare (NIH\&FW), All India Institute of Public Health Kolkata (AIPH) etc. and Institutes affiliated to All India Colleges of Technical education (AICTE). Some Students may pursue an MPH or an equivalent degree online also through the worldwide Programs in Public Health, Medical Schools, Schools of Public Health, and Schools of Public Affairs [4].

University Grants Commission (UGC) in India expects every teacher to participate in teaching, which may include any or all the lectures, tutorials, laboratory sessions, seminars, fieldwork, projects and other such activities. Every teacher is supposed to give general assistance to students in removing their academic difficulties; and participate in the invigilation and evaluation work connected with tests / examinations; and take part in extra-curricular, co-curricular and institutional support activities as required. The workload of a teacher shall consider activities such as teaching, research and extension, preparation of lessons, evaluation of assignments and term papers, supervision of fieldwork as also guidance of project work done by the students. The time spent on extension work, if it forms an integral part of the prescribed course, shall count towards the teaching load [3].

It is with this background that this author had published his first article on the issue of public health education titled "Influencing Public Health without authority" in 2012 [4] and the present study aimed to update the attention given to the capacity development in our public health schools. The background and theory supporting this research, is to strengthen the importance of such study and provide a factual background, clearly defined problem, proposed solution to move forwards. A brief literature survey was done, and the scope and justification of the progress achieved is justified.

\section{Materials \& Methods}

\section{Study Setting}

I. This study is a qualitative interpretive and ethnographic case study conducted by the author and HOD of the MPH school or the autonomous universities /institutions visited, and Departments of Community medicine/ Preventive and Social Medicine of the Medical Colleges visited.

II. The opportunities included a: visits as a guest faculty for specific modules in MPH schools; b: As Key facilitator of one week 's domain specific \{Filed epidemiology, State specific strategic analysis for investment in Health, 
Integrated management of neonatal \& childhood illnesses (IMNCI), Research Methodologies, Role of Biostatistics in Public Health etc.\} workshops; C: Interviews of the candidates for the faculties of MPH schools, as a chairperson or member of the recruitment committees

\section{Sampling \& Sample Size}

There was no sampling involved. The observations and discussions were made in the institutions visited by the author during his visit for various professional demands over a period of 8 years. It could best be called opportunistic in nature, The study was conducted in 20 institutions (IIHMR Jaipur \& Bangalore, PHFI Gurgaon, PGI Chandigarh, National Institute of Health and Family Welfare- New Delhi, National Centre for Diseases Control- Delhi, Mahatma Gandhi Institute of Medical Sciences- Wardha, Government Medical CollegeNagpur, BJ Medical College-Pune, Grant Medical CollegeMumbai, MS Ramaiah Medical College- Bangalore, KSRDPRU Gadag, RGIPH-Bengaluru, PGI Bangalore, JNMC Davangere, Yenopiya MC Mangaluru, BLDEA College Vijaypura, MS Rampure Medical College Kalburgi, Kalburgi Institute of Medical Sciences (KIMS) distributed across major states offering MPH courses except north-eastern part of the country over a period of last 8 years since my last study published. Majority of these visits were repeat visits to the same institutions and changes observed over the previous visits were noted.

\section{Data Collection Methodology}

The methodology included mostly formal interactions with key stakeholders (Heads of the course) of the MPH / MD courses, a few informal interviews some of their faculty members. Informal interactions also included hundreds of scholars and a few products of such schools in their worksites met during my professional consultancies across the country. The evaluation process included: a: interviews of the local focal points (coordinators) of MPH courses on training methodologies like Pedagogical lecture, journal clubs, tutorials, demonstration of equipment, field epidemiology, practical exposures like visiting local health offices, participation in monitoring national health programs, field epidemiology of investigating local disease outbreaks, shadowing health functionaries etc b: observation of operational research opportunities and guidance to take up such researches and shadowing health functionaries c: Examination process including the practical's like medicosocial case studies of IMNCI, Maternal care, Communicable diseases -Malaria, TB, Dengue, Chikungunya, Industrial safety and non-communicable diseases like Diabetes, Hypertension and Cancers $\mathrm{d}$ : community transaction, mapping and strategic analysis for investment in health e: identifying and placement in right types of institutions for internship and f: arrangements for oversight of mandatory internship f: their Publications (as mandated by UGC) of papers by the faculty g: Continuing the research or follow up of their PG dissertations on job g) assessing the readiness of the candidates to take up the tasks assigned in their first jobs, and the role their institute had played in their empowering for the challenges of the need of the job market.

\section{Data Analysis}

At best this data analysis can be considered as SWOT analysis by the author in consultation with local leads, as it is mainly qualitative in nature. The focus is on assessing the situation, limitations and their reasons for going beyond syllabus. Quantification is not attempted as the duration of the data is spread over 8 years. It is also true that the situation has not changed drastically even in long standing MPH schools.

\section{Results}

Between 1997 and 2016-2019, the number of institutions offering MPH programmes increased from 2 to 60. There are at least another 10 such schools that are not listed so far on any website. Some of the colleges that started as Public Health schools 15 years ago have migrated to call themselves as Colleges of Health and Hospital Administration or Hospital administration offering MHA, MBA or MD in Hospital Administration. There is also a recent UGC guideline to call courses affiliated to any recognized university to give MBA degree and those affiliated to All India Council of Technical Education (AICTE) to give Post Graduate Diploma in Health/Hospital Management (PGDHM). All programmes include some field experience, but none can claim to be adequate to produce skilled candidates to take up any public health task independently after the completion of the course. The ratio of faculty number to students enrolled ranged from 1: 1 to 1:20. However no institution can claim having all the super speciality expertise among their own faculty.

Currently, several Indian institutions and autonomous universities, Health Universities and a few national public health institutions offer the Master of Public Health (MPH) programme. I classify these institutions in four categories: Cat-1 Nationally recognised Government institutions and Private Institutions with Government representatives on their governing board e.g. NCDC, NIHFW, NIE, AIIMS, AIH\&PH, IIHMR, PHFI etc. Cat-2) State governments or state government sponsored institutes or State Health University or RDPR Universities under the University Grants Commission (UGC) Cat-3, AICTE recognized universities etc. Cat-4. Public Health Schools under Autonomous Universities. The quality of the training is widely varied from institutions 
to institutions, based on the above categories. The course fee ranges from 32000 to 200,000 per year as we enter 2020 . The present status can be described as:

\section{Strengths}

Governance: Government sector institutes and large private institutions having influential persons on their governing board have an advantage of access to public health system to expose the scholars to experimentations, field work and doing internship. They also manage to get government and even international projects where in their faculty and students get adequate exposure to public health practices. The institutions under medical education departments or Health universities are better off in preparing the scholars for teaching and research. On the other hand, institutions under department of Health services have the distinct advantage of exposing their scholars in health policy, programming, implementation, monitoring and evaluation and interacting with community. Very few institutions run under the department of Rural development and Panchayat Raj universities have distinct advantage of exposing the scholars to Rural /urban immersions to understand better the Sociocultural aspects of health programming. Since the primary health care institutes are under the administrative control of Zilla Parishad, the scholars are at advantage of learning about nitty-gritties of comprehensive primary health care.

Physical Infrastructure: Most of category $1 \& 2$ institutions have good infrastructure as they are existing for more than a decade and are funded by government or Big private sector for profit organizations. They do link with Government and Private Health Industry, Municipal authorities etc.

Faculty: i) The national specialized institutions like NCDC, NIHFW, NIE, AIH\&PH have large number of senior staff with lots of experience in their field of specialization, access to field programs and prepare the scholars very well in their respective areas. ii) The large private sector institutions have adequately trained, (some of them even in foreign countries) staff. They are equipped with eloquent pedagogical teachers and or Researchers to train their scholars in what I call "Computer based public health professionals", good for Research and training. Some of these international degree / certificate holders find it difficult to empower their students under local working conditions as they themselves have not worked under such situation. A few of them do learn on the field and quickly adjust but majority fail and remain good speakers iii) Institutions governed by University Grant Commission have the option of appointing guest professors drawn from teaching of public health practicing professionals to compensate for the limited in-house expertise. These institutions depend on senior external domain experts as the UGC has specific provision for the domain specific experts from academic side or field or practicing professionals. UGC allows such experts to be brought in as Emeritus Professors, Honorary Professors or Visiting professors etc with maximum cap of INR 50,000 per month, and not more than 4 hours per day at a remuneration of INR 1500 per hour. Most of the new institutions are making good use of this opportunity.

Other Resources: Funding either by the state or the Institution matters most in routine field visits for learning, community projects and building rapport with the community around to get support for their community activity. Category $1 \& 2$ institutions don't have much problem for resources.

\section{Weaknesses}

Internal factors like infrastructure, buildings, classrooms and hostel facilities, libraries, sports facilities, transport for field visits and attachment with local hospitals, committed and motivated faculty are some of the weaknesses. Limited capacities to get research projects, lack of provision for getting domain experts visiting faculty and poor Industry collaboration are the other threats.

Governance: Autonomous universities depend upon their governing bodies most of whom are running medical colleges, and their MPH schools are run under the banner of Allied Sciences. In the larger canvas MPH courses may not get due importance from the management. The filed activities are restricted to their field practice areas and scholar's exposure to larger program initiatives are generally weak and depend upon the local level coordination with the government authorities, that may change every time the local authority changes.

Physical Infrastructure: Category 2 institutions are mostly new and are building up the infrastructure though the progress is slow. Some of them share facilities like library, vehicles etc. that may sometime pose a challenge for planning. Category 3 and 4 institutions are run in an existing composite complex and may not have adequate spaces and manage with other courses.

Faculty: Newly opened Category $3 \& 4$ Public health schools either under autonomous universities or institutions under AICTE generally manage with skeleton newly recruited inexperienced staff. Their in-house faculty are young with no experience and find it difficult to guide scholars be it for public health practice or dissertations or planning, monitoring and evaluating health system performances. Some of them do seek help of local health authorities for exposure to national health programs, but never recognize them as teachers or faculty. For me the UGC rules of considering 2 hours of the practical sessions equivalent to one hour of theory appears to be detrimental for exposing 
the scholars to practical exposure. Faculty generally opt for pedagogical teaching than spending time in the field. In one for the interview for recruiting the teaching staff, the author came across 3 of the five candidates shortlisted from the same institution for research associate position. They had 1-3 years teaching experience in the parent institute from where they had acquired their MPH, but surprisingly neither during the studentship nor as a faculty had gone even once to investigate any outbreak in the field. Naturally post-graduates from such institution may excel in theory but fail to meet the demand of empowering the scholars for the health services.

Other Resources: Category 2 \& 3 institutions run on skeleton budget and therefore scholars are hardly exposed any filed activity, except in colonies or slums around the institution.

\section{Discussions}

\section{Literature Review}

The Master of Public Health (M.P.H.) is a multi-disciplinary professional degree awarded for studies in areas related to public health. The MPH degree focuses on public health practice, in contrast to MD (P\&SM, Community Medicine, Public Health etc.) and Doctor of Public Health (Dr.P.H.) or $\mathrm{Ph}$. D in Public Health that give prominence to research or teaching. In some countries the MPH degree program is only available for medical graduates (MBBS or equivalent), those without the medical degree can join the Master of Science in Public Health (MSPH) program. The MSPH degree is an academic public health degree rather than a professional public health degree. It is more research-oriented than the MPH degree and the scholars must complete a research thesis/project of publishable quality. In the UK a master's in public health, Global Health or International Health all concentrate on improving the general health of the population of both individual countries and communities and the world. Entry into these postgraduate courses requires either an undergraduate medical degree or a degree in a subject with a strong bioscience's emphasis [6].

Master of Public Health programs are available in India through Medical Colleges, Public Health Schools, Colleges of Allied Sciences and specialized National institutions of Public Health like National Institute of Mental Health and Neurosciences (NIMHANS) Bengaluru, National Institute of Epidemiology, Chennai(NIE), National Centre for Disease Control (NCDC) Delhi, National Institute of Health \& Family Welfare (NIH\&FW), All India Institute of Public Health Kolkata (AIPH) etc. and Institutes affiliated to All India Colleges of Technical education (AICTE). Some Students may pursue an MPH or an equivalent degree online also through the worldwide Programs in Public Health, Medical Schools,
Schools of Public Health, and Schools of Public Affairs [4].

When studying for an MPH degree scholar is expected to develop an integrated interdisciplinary, cross-cutting set of overall competency in domains like Health and Hospital management, Communication and Informatics, Diversity and Culture, Rural / urban Immersion, Leadership, Professionalism, Program Planning, Epidemiology, National Health Programs, Health System, National Health Policy, International health Public Health Biology, and Systems Thinking.

Genesis of Public Health in India: The high mortality among the British and Indian troops operating in India attracted the attention of British Parliament which set up a Royal Commission in 1860 to visit India. The commission, realizing that the state of health of civilian population was a danger to the troops made recommendations for recruiting sanitary commissioners in three major provinces-Bengal, Madras and Bombay, following that the first chair of Professor of Hygiene was established in the Calcutta Medical College in 1865 [5]. The devastating epidemic of plague in 1896 led to the establishment of Indian Plague Commission. It resolved that the Medical Officers of Health should have a diploma in public health as a special qualification to discharge their duties successfully. Based on the recommendation of the Plague Commission, Calcutta University decided to initiate the Diploma in Public Health8. The Governments of India and Bengal with the aid from charities established the School of Tropical Medicine (STM) in Calcutta in 1920, however soon STM expressed difficulties in continuing with the DPH training. Dr. W S Carter on behalf of Rockefeller Foundation helped build and equip an Institute, that was named All India Institute of Hygiene \& Public Health (AII\&PH) was formally opened on 30th December 1932 by John Anderson, Governor of Bengal with Lt. Col. A D Stewart as its first Director [6].

The Institute started with four sections-Public Health Administration, Malariology \& Rural Hygiene, Vital Statistics \& Epidemiology, and Biochemistry \& Nutrition. Shortly, the fifth section of Maternity \& Child Welfare was added with the help of Countess of Dufferin Fund. The year 1934 saw the establishment of the section of Sanitary Engineering. Post- World War reconstruction plans required immediate industrialization, focussing attention on health of industrial workers and in 1945 Industrial Hygiene was added as another section. Increasing need of application of bio-metric methods in analysing data - both research and routine, required biostatics to be separated from Epidemiology and become a separate section. During this period the Institute offered three courses - Diploma in Public Health (DPH) and Diploma in Maternity and Child Welfare (DMCW) for medical graduates and Diploma in Public Health and Hygiene (DPH \& Hy) for medical licentiate. Majority of the states depended 
upon training of their in-service medical officers until 1975.

Traditionally in India, medical colleges were the centres for creating public health professionals6. In the last two decades, there has been a significant change in the way public health professionals are trained in the country. There has been a conscious shift towards the creation of schools of public health outside medical colleges, allowing non-medical personnel to acquire academic competencies in public health disciplines. Currently the Master of Public Health (MPH) programme is being offered by various institutions and universities under the minimum standards for a master's degree laid down by University Grants Commission regulations, 20035. In the absence of a formal body or council for regulating public health education, to date, there has been limited information on evolution, development and issues related to MPH programmes in India.

The first MPH programme in India was launched at Mahatma Gandhi University, Kottayam, Kerala, in 1995. This was followed by the Achutha Menon Centre for Health Science Studies, Sree Chitra Tirunal Institute for Medical Sciences and Technology, Kerala in 1997. During the decade from 1997 to 2006, four institutions commenced MPH programmesSam Higginbottom Institute of Agriculture, Technology and Sciences, Allahabad in 2000; the National Centre for Disease Control, New Delhi in 2005; and both Jawaharlal Nehru University, New Delhi and the Interdisciplinary School of Health Sciences, University of Pune, Pune in 2007 [6].

Since the beginning of 21st Century in India anyone with an accredited undergraduate degree, may pursue an MPH / DPH /PGDHM/ master's in health administration (MHA), MD in Hospital Administration degree. Based on the accreditation criteria of the Medical Council of India Public Health (MCI) an MPH or any of the degrees listed are not a clinical degree. With the beginning of the public health course in the new nomenclature of MPH, the DPH course have also been renamed.

An MPH Degree must prepare medical professionals who can run community health facilities, national health programs, state and district organizations, NGOs, and International health efforts, as well as oversee nursing departments. It's one of the fastest growing areas of medical administration.

Public health jobs involve environmental science, biosecurity, epidemiology, disease specialist, public health education, disease analysis, community activism, sanitarian, food service, hazardous waste inspection and control, administration, legislation, law, and coordination. All these areas of concentration can be built during one's career in public health. Having knowledge and the specialty in any of these areas will lead to greater impact and higher paying careers.

MPH graduates have opportunities in the health system and academic institutions and jobs in academic and research institutions are increasing in the last decade. Non-academic jobs are in healthcare delivery, monitoring and evaluation, health Insurance and Health financing. There are significant challenges for entry into the public and private health sector, which are due to lack of Institutionalising public health service at central, state and district levels, requisite skills among the scholars, uncertain recruitment processes, poor working conditions, poor living conditions in rural areas, lack of competitive salaries, unclear expectations of work roles and feedback [2].

Flawless \& speedy learning is the need of modern knowledge era. This requires institutionalized attempts to facilitate life- long learning by adopting an education system, which is learning oriented rather than teaching or a university degree oriented. The central task of MPH education institutions is to implant a will and provide facilities for learning. The effective learning needs a simultaneous activation of three dimensions viz. content, incentive and interaction. The students doing MPH today need to be groomed to face new scenarios, derive meaningful and sustainable solutions by partnering with practicing $\mathrm{PH}$ persons. A learning-oriented education system should ensure that the interactions are devised and continuously assessed for their ability to engage the learners emotionally. Impart the contents of learning with clarity authenticity, applied aspects and hands on experience. Engaging learners in experimentation, experience sharing projects, stepping into health professionals' roles and similar activities as they lead to development of relatively clear and permanent neural traces in the brain. Institutions should also infuse the habit of professional and personal development [6].

The Ministry of Health and Family Welfare has prepared a model course curriculum for master's in public health (MPH). The course is designed to be a two years' fulltime program including internship and dissertation. The course intends to develop the analytical and assessment skills of candidates for collecting and interpreting information in areas of policy planning, communication, advocacy and evaluation of public health data and financial planning, leadership and management skills for running public health programs in the country [7]. UGC has recommended adopting the course curriculum in all universities and conveying relevant instructions to all the affiliated colleges / institutes on $18^{\text {th }}$ September 2018. Most of the new schools have either adopted in toto or adapting with minor modifications. Newer learning and teaching methods developed by University Grants Commission (UGC) Informal teaching and learning methods 
are being adapted by some of the public health schools [3]. The rapid expansion of institutions offering has created a gap in training in general and in actual skill building in majority of the institutions. MPH programmes being offered in India have witnessed a rapid expansion in the past two decades. Nearly sixty institutions in India offer MPH programs as of January 2020 and three quarters of MPH graduates are from a non-medical background. This growth in supply of public health graduates is not yet matched by an increased demand. Despite the recognized need to strengthen the public health workforce in India, there is no clearly defined career pathway for MPH graduates in the national public health infrastructure. Public Health teaching Institutions and public health bodies and Industries must collaborate to design and deliver MPH programmes to overcome the lack of skills among the scholars that would influence the national and state governments to create appropriate positions in the Public Health System.

Economy of the health Industry is growing in India as one is seeing in the last 5 years the number of public health school's expansion is phenomenal and the demand for the skilled professionals. Public Health Career opportunities are expanding in the recent years [10].

1. Management Policy Advisor/ Consultant: One of the most direct ways to put your master's degree in public health is to manage policy from a government, corporate or non-profit entity. MPH will allow a policy advisor or manager to take on an executive role. An advisor / Consultant is expected to have a deep understanding of public policy, organization, administration, and legislation. Some medical graduates seek a master's in public health or MD in Community Medicine/Public Health in order to supplement their medical degrees. This makes them a field expert from both a micro and macro level. They have a deep understanding of illness and treatment, as well as how to bring the best treatments to entire groups and communities.

2. Tropical Disease Specialist: A tropical disease specialist is an expert in diseases that are found in tropical parts of the world requiring a background in biology, medicine, politics, and disease as well as a master's degree in public health. In order to prevent spread, transmission and begin processing administration of vaccines and cures, a tropical disease specialist must have a keen understanding of public health organization and administration. They'll take samples from the local environment as well as tissue samples from community members to find the right vaccine and cures for the disease that is found in that area. If a tropical disease specialist chooses to work in an urban area.
3. A reproductive health specialist is vital to all communities, as it involves the very source of growing a population, especially the community's most vulnerable groups-mothers and children. They must understand prevalent diseases related to reproductive health such as HIV/AIDS and STDs. Prior experience in health system or training in medical speciality will go a longways.

4. Public Health Engineer: Public health engineers are primarily concerned with the public design and setting that might influence the community's health. These engineers have a background in the industry, infrastructure, machines, equipment, cold chain equipment, Solar energy the environment, and basic construction of toilets, water filter beds etc. They know how to design a setting that would reduce public infection, injury or illness that occurs due to the environment.

5. NGO/CSR Director: A nongovernmental organization (NGO) or Corporate Social Responsibility is a crucial part of the public health fabric. NGOs have a tremendous influence on communities in need because they get directly involved in communities that government policy might not be able to reach. NGOs' initiatives have a great impact on public health and administration. An NGO director will have an influence on all tasks and actions that the organization has in a group.

6. Health Centre/Hospital Administrator: A health centre or hospital administrator is essential to the functions of public health. When administering health care, treatments, cures, etc., they need to coordinate large groups to organize services. A health centre administrator will usually work at a hospital. The work of a health care administrator usually involves handling paperwork, forms, and liaising between doctors, nurses, healthcare companies, insurance companies, and clinics. Their work is extremely important as their job requires handling sensitive medical information. If an important detail is missing, there may be huge consequences.

7. Education for health care administrators requires a minimum of a bachelor's degree. A master's degree boosts one's knowledge on business, health services, and health care. On average, a health care administrator makes approximately $\$ 84,000$ and up to or over $\$ 144,000$ per year. This average is bound to go up in the next five years.

8. Biostatistician: Biostatisticians collect relevant information, numbers and data for researching public health, environmental science, and medicine. Biostatisticians are essential to organization and administration of public health work. Without it, there would be no way to understand how certain diseases are 
impacting communities and whether the budget spent on treatments are influencing the groups effectively.

9. Legislative Policy Advisor: A legislative policy advisor is a legal and medical expert who uses his or her expertise to influence political organizations, campaigns or politicians. Their work requires extensive research, keeping up to date with current policies, a keen understanding of pharmaceuticals, medical industry, insurance and public health.

10. Outcomes Researcher: An outcomes researcher handles analysing the outcome of any health care practice or program. They collect data from health care facilities, NGOs, corporations, government agencies and other sources where healthcare programs and practices are implemented. Outcomes researchers work at hospitals and other health care facilities. Their work involves analysing budgets, public health issues, statistics, and medical practice. Having a bachelor's degree in math, stats, sociology and anthropology helps become an effective outcomes researcher, but having a master's degree in public health will help a candidate make a larger salary and take on executive positions.

The principle of never stop learning goes a long way to be a successful teacher. The classroom is a dynamic environment, bringing together students from different backgrounds with various abilities and personalities. Being an effective teacher therefore requires the implementation of creative and innovative teaching strategies in order to meet students' individual needs. Some of the newer techniques are listed below: $[5,6]$.

1. Visualization: Bring dull academic concepts to life with visual and practical learning experiences, helping your students to understand how their schooling applies in the real-world. Examples include using the interactive whiteboard to display photos, audio clips and videos, as well as encouraging your students to get out of their seats with classroom experiments and local field trips [10].

2. Cooperative learning: Encourage students of mixed abilities to work together by promoting small group or whole class activities. Through verbally expressing their ideas and responding to others your students will develop their self-confidence, as well as enhance their communication and critical thinking skills which are vital throughout life. Solving mathematical puzzles, fictious outbreak situations, conducting scientific experiments and acting out short drama sketches are just a few examples of how cooperative learning can be incorporated into classroom lessons.

3. Inquiry-based instruction: Pose thought-provoking questions which inspire your students to think for themselves and become more independent learners. Encouraging students to ask questions and investigate their own ideas helps improve their problem-solving skills as well as gain a deeper understanding of academic concepts. Both of which are important life skills.

4. Differentiation: Differentiate teaching by allocating tasks based on students' abilities, to ensure no one gets left behind. Assigning classroom activities according to students' unique learning needs means individuals with higher academic capabilities are stretched and those who are struggling get the appropriate support. This can involve handing out worksheets that vary in complexity to different groups of students or setting up a range of workstations around the classroom which contain an assortment of tasks for students to choose from.

5. Technology in the classroom: Incorporating technology into teaching is a great way to actively engage students, especially as digital media surrounds young people in the 21st century. Mobile devices, such as iPads and/ or tablets or even simple phones, can be used in the classroom for students to record results, take photos/ videos or simply as a behaviour management technique.

6. Behaviour management: Implementing an effective behaviour management strategy is crucial to gain students respect and ensure students have an equal chance of reaching their full potential. Examples 'Golden time' can also work for students of all ages, with a choice of various activities such as games or no homework in reward for their hard work.

7. Professional development: Engaging in regular professional development programmes is a great way to enhance teaching and learning in classroom. Sessions can include shadowing / working as health functionaries, participating in surveys, monitoring national /state health initiatives etc.

\section{Threats}

Complex health care system of the country coupled with our education's limitation to prepare our scholars for daunting challenges, difficult emotional or social experiences are the major threats. lack of Institutionalising public health service at central, state and district levels, quoting lack of requisite skills among the scholars, uncertain recruitment processes, poor working conditions, poor living conditions in rural areas, lack of competitive salaries, unclear expectations of work roles may take away the sheen from the MPH courses in the years to come. We have already witnessed about 10$20 \%$ of seats going vacant in Private institutions with high course fees. Sudden increase in the number of institutions offering MPH, the availability of experience staff is already a threat. Since most of the institutes are recruiting staff on contractual basis the career progression is uncertain 
and therefore institutions located out of state capitals are facing much larger threat. Few Government institutes who give stipend or honorarium may survive the rest will find it difficult get students.

\section{Conclusive Summary}

Public Health management (MPH) students are not required to confine themselves to conventional epidemiology, National Health Programs, management, finance, insurance or corporate sector. Today, Ayushman Bharat, Universal Health Coverage, SDGs, Client centric approach to health and hospital business, Health Apps and biotechnology are emerging as potential employers. With a lot more avenues opening, there is this need for grooming students for these new age fields, which require certain expertise and compatible skill set. Obviously, the traditional approach is not going to help in this regard. It is imperative for today's students to acquire the skillsets that are extremely essential in the changing academic and business environment.

Counselling and mentoring play a crucial role here. For students, it may be a touch difficult to zero in on a skill set that would stand them in good stead. Right coaching and mentoring, therefore, can come in handy. The experts need to assess and evaluate students' precise needs so that they can take necessary steps. With evolving market dynamics, academicians feel that Public Health schools in the country should be more instrumental in helping students understand the changing game of business and shaping entrepreneurial mind-set. Let students learn to handle the business situations in a realistic manner. Students must get their hands soiled into such situations. Their dissertation should be an outcome of their business experience and genuine findings, and it should not be just theoretical submission. In this context it is imperative for MPH schools to anticipate these trends and prepare managers with the evolving concepts of Public Health business. Industry academia interface is the best solution to bridge the widening skills gap. It is high time we did away with parroting textbooks.

More and more MPH schools are now focusing on increased industry-academics interaction. Through various programmes, efforts are made to enable students rub shoulders with experts in their respective fields so that they can pick the latter's brains in their bid to pursue success.

Through management programs, MPH schools should motivate the students to think as an entrepreneur who can identify and evaluate opportunities especially those that emerging technologies bring, and new markets create. While the capacity to solve linear cause and effect problems is essential, more crucial is the development of adaptive capacities. It is possible to groom the rich talent pool in our country for shouldering the responsibility of scaling newer heights in Health and Hospital business.

\section{Way Forwards}

To address the widening skill-gap, the MPH course globally more specifically in India need to ensure:

1. An exact survey under the joint leadership of UGC, MOH\&FW and Private Health Industry to pinpoint the right skills with which Public Health schools should equip their students be taken up urgently

2. UGC \& MOH\&FW need to define minimum skillsets that the final exit examination of each institution should ensure before awarding the degree. E.g. Field epidemiology for investigating a communicable diseases outbreak, Screening and Counselling skill for NCDs, and occupational diseases, skills for Medico-social case studies to take the lessons from individual case to community interventions, Village and Urban health determinants and the interventions to mitigate the health problems due to environment degradation and disasters etc.

3. All institutions should expose their scholars to the publicrural and urban health and private health industry for at least one third of the duration of the course to introduce them to latest trends in the program/health facilities planning, implementation, monitoring and management syllabus.

4. Alliances shall be of a great help; therefore, a national oversight authority be created to ensure better coordination among MPH schools, other health academic institutions, Public and Private health facilities, National and State health programs for shadowing experience

5. Faculty training programs should be designed and popularized so that they know the ground reality and plan to expose students directly.

6. Lastly, Syllabus must incorporate latest industry trends and must be upgraded continuously.

\section{References}

1. Mishra S, Education for the Masses: Lessons from Gandhian Praxis, Suresh Mishra, Articles: On and By Gandhi.

2. Henrietta H, Fore ED (2018) UNICEF's culture and Living our core values.

3. Prakash V (2003) UGC (Minimum Standards of Instruction for the Grant of the master's degree through Formal Education) Regulations, 2003.

4. Suresh (2012) Influencing Public Health without Authority. Indian Journal of Public Health 56(1): 22-30. 
5. (2019) Fostering Social Responsibility and community engagement in Higher education institutions in India. National Curriculum Framework and guidelines, pp: 1-26.

6. Negandhi H, Sharma K, Zodpey SP (2010) How can departments of community medicine shape the future of Public Health Education in India?. Indian J Public Health 54(4): 184-189.

7. A Model course curriculum for master's in public health (MPH).
8. Tiwari R, Negandhi H, Zodpey S (2018) Current status of Master of Public Health programmes in India: a scoping review. WHO South-East Asia J Public Health 7: 29-35.

9. Masbah S, Mahboob U (2017) Strengths, weaknesses, opportunities, and threats analysis of integrating the World Health Organization patient safety curriculum a qualitative case study. J Educ Eval Health Prof 14: 35.

10. Quazi Syed Z (2013) Career Opportunities for Master of Public Health Graduates in India. Asia Pacific Journal of Health Management 8: 1. 